\title{
Endokrine Resistenz beim Mammakarzinom überwinden
}

Selektive Inhibitoren der Cyclin-abhängigen Kinasen 4 und 6 (CDK4/6) wirken proliferationshemmend und sollen in der Kombination mit einer endokrinen Therapie die Entstehung einer endokrinen Resistenz vermeiden. Das konnten Daten der Phase-III-Studie MONARCH-2 bestätigen.

n der doppelblinden Studie wurde bei Patientinnen mit fortgeschrittenem Hormonrezeptor(HR)-positivem und HER2-negativem Mammakarzinom Wirksamkeit und Sicherheit des selektiven CDK4/6-Hemmers Abemaciclib in Kombination mit Fulvestrant mit einer Fulvestrant-Monotherapie verglichen. Für die Teilnahme mussten die Patientinnen einen Progress erlitten haben nach einer vorangegangenen neoadjuvanten oder adjuvanten enokrinen Therapie (ET) innerhalb von weniger als 12 Monaten oder unter einer ET in der Erstlinie bei einer metastasierten Erkrankung.

Die Patientinnen erhielten im Verhältnis 2:1 kontinuierlich Abemaciclib (AF; 150 mg 2-mal täglich; $\mathrm{n}=446$ ) oder Placebo $(\mathrm{n}=223)$ und Fulvestrant $(\mathrm{F}$;
$500 \mathrm{mg}$ i.m. an Tag 1 und 15 des 1. Zyklus und anschließend alle 4 Wochen).

Die AF-Kombination war F plus Placebo beim primären Endpunkt progressionsfreies Überleben (PFS) überlegen (median 16,4 vs. 9,3 Monate; Hazard Ratio [HR] 0,553; $95 \%$-Konfidenzintervall [95\%-KI] 0,449-0,681; p < 0,001). Die Gesamtansprechrate (ORR) war im Interventionsarm mehr als doppelt so hoch wie im Kontrollarm (35,2 vs. 16,1\%). Bei Patientinnen mit messbarer Erkrankung war dieser Unterschied noch ausgeprägter (48,1 vs. $21,3 \%$ ). $3,5 \%$ der Patientinnen dieser Subgruppe erreichten unter der Abemaciclib-Kombination eine komplette Remission.

Die erhöhte Wirksamkeit ging mit mehr Nebenwirkungen einher. Die Rate an Diarrhöen lag im AF-Arm bei 86,4 \% im Vergleich zu 24,7\% im Kontrollarm, Neutropenien traten bei $46,0 \%$ der Patientinnen unter $\mathrm{AF}$ und $4,0 \%$ unter $\mathrm{F}$ plus Placebo auf, Nausea bei $45,1 \%$ und $22,9 \%$, Fatigue bei $39,9 \%$ und $26,9 \%$. Im AF-Arm brachen 15,9\% der Teilnehmerinnen die Therapie aufgrund inakzeptabler Nebenwirkungen ab, im Standardarm 3,1\%.

Fazit: Abemaciclib in einer Dosis von 150 mg 2-mal täglich zusätzlich zu Fulvestrant resultiert in einem verlängerten PFS und einer höheren ORR bei Frauen mit HR-positivem und HER-2-negativem Mammakarzinom und einem Progress unter einer ET. Das Toxizitätsprofil entspricht in etwa dem von anderen CDK4/6-Inhibitoren. Friederike Klein

Sledge GW et al. MONARCH 2: Abemaciclib in Combination With Fulvestrant in Women With HR+/HER2- Advanced Breast Cancer Who Had Progressed While Receiving Endocrine Therapy. J Clin Oncol. 2017. Jun 3 [Epub ahead of print].

\section{Eingeschränkte Rolle von Capecitabin beim frühen Hochrisiko-Mammakarzinom}

\begin{abstract}
Trotz Intensivierung der adjuvanten Chemotherapie beim frühen HochrisikoMammakarzinom bleibt die Rezidivrate bislang mit mehr als $20 \%$ über 5 Jahre hoch. Die Rolle von Capecitabin in dieser Situation ist unklar. In einer Metaanalyse randomisiert-kontrollierter Studien ging man dem jetzt nach.
\end{abstract}

\footnotetext{
A usgewertet wurden Studien, in denen eine Standardchemotherapie mit und ohne Capecitabin in der neoadjuvanten oder adjuvanten Situation verglichen wurde. Für die Metaanalyse wurden die Daten von 9.302 Patientinnen aus 8 Studien extrahiert und die Wahrscheinlichkeiten im Beobachtungszeitraum (Hazard Ratios, HR) für das krankheitsfreie Überleben (DFS) und das Gesamtüberleben (OS) sowie die relative $\mathrm{Er}$ eigniswahrscheinlichkeit (Odds Ratio, OR) für Toxizitäten gepoolt verglichen. In Subgruppenanalysen wurden Patientinnen mit triple-negativem (TNBC) und nicht triple-negativem Mammakarzi-
}

nom sowie die Therapie mit Capecitabin zusätzlich oder als Ersatz zur Standardchemotherapie gegenüber gestellt.

Insgesamt zeigte sich kein Einfluss von Capecitabin auf das DFS (HR 0,99; $\mathrm{p}=0,93)$ oder das OS (HR 0,$90 ; \mathrm{p}=0,36)$. Ein DFS-Vorteil war allerdings erkennbar, wenn nur die Studien ausgewertet wurden, bei denen Capecitabin zu einem Chemotherapieregime hinzugenommen wurde, nicht aber diejenigen, in denen es eine Standardchemotherapie ersetzte (HR 0,92 vs. 1,$\left.62 ; \mathrm{p}_{\text {Interaktion }}=0,002\right)$. Am ehesten spielt Capecitabin eine Rolle beim TNBC: Die Hinzunahme zur Standardchemotherapie resultierte in einem signifikanten Vorteil gegenüber der alleinigen Standardtherapie (HR 0,72 vs. 1,$\left.01 ; \mathrm{p}_{\text {Interaktion }}=0,02\right)$. Die Metaregressionsanalyse zeigte auch ein verbessertes OS in Studien mit einem hohen Anteil von Patientinnen mit TNBC (Regressionskoeffizient $\mathrm{R}=-0,967 ; \mathrm{p}=0,007$ ).

Die Therapie mit Capecitabin führte allerdings auch mehr als doppelt so häufig zu Diarrhöen vom Grad 3/4 (OR 2,33; $\mathrm{p}<0,001)$ und 13-mal so häufig zu einem Hand-Fuß-Syndrom vom Grad 3/4 (OR 13,23; $p<0,001)$. Fast 4 -mal so häufig wurde eine Capecitabin-Therapie wegen der Nebenwirkungen abgebrochen (OR $3,80 ; \mathrm{p}<0,001)$.

Fazit: Wegen der Effekte auf DFS und OS fordern die Forscher eine Berücksichtigung von Capecitabin zur Therapie des TNBC, auch wenn die Toxizität durch die bekannten Nebenwirkungen unter seiner Hinzunahme ansteigt. Friederike Klein

Natori A et al. Capecitabine in early breast cancer: A meta-analysis of randomised controlled trials. Eur J Cancer. 2017;77:40-7. 